Commun. Korean Math. Soc. 17 (2002), No. 2, pp. 331-347

\title{
GEOMETRIC CONIC SPLINE APPROXIMATION IN CAGD
}

\author{
YOUNG JOON AHN
}

\begin{abstract}
We characterize the best geometric conic approximation to regular plane curve and verify its uniqueness. Our characterization for the best geometric conic approximation can be applied to degree reduction, offset curve approximation or convolution curve approximation which are very frequently occurred in CAGD(Computer Aided Geometric Design). We also present the numerical results for these applications.
\end{abstract}

\section{Introduction}

To approximate a given plane curve by a polynomial or by a rational curve is one of the most frequently occurring problems in CAGD. This problem arises from any process converting the curves, such as the intersection curves of two surfaces, polynomials of high degree, offset curves or convolution curves, etc., into an actual CAD system. Furthermore, all conversion problems which cannot be solved exactly invoke such an approximation problem.

Thus many efforts and proposals for dealing with these kinds of problems have been made in the recent twenty years or so. Most publications focus on certain aspects, e.g., conversion problems $[3,18,20]$, computing offsets $[2,12,14,15]$ or high accurate geometric approximations $[1,9,16,24]$. Also, Eisele $[11]$ characterized the locally best geometric approximation to a given regular plane curve $\mathbf{c}(s)$ by a polynomial curve $\mathbf{b}(t)$ with arbitrary degree $d$ and with all possible contact order $k$ at both end points of two curves $\mathbf{c}(s)$ and $\mathbf{b}(t)$.

Received July 2, 2001.

2000 Mathematics Subject Classification: 41A50, 41A52, 65D05, 64D07.

Key words and phrases: conic spline, Chebyshev approximation, geometric Hermite interpolation, offset curve, convolution curve.

This work was supported by Korea Research Foundation Grant (KRF-2000-003D00027). 
The conic spline which is also called quadratic rational $B$-spline is one of the most widely used curves in industry, e.g., to design the bodies of aircraft, to design outlines of fonts $[2,21,22]$ or to express circular arcs [19] in CAD systems. In this paper we characterize the best geometric conic spline approximation to regular plane curve, which means that the ( $L_{\infty}$-sense) 'best' approximation to regular plane curve by the conic curve with all possible contact order at both end points of two curves is characterized. We also prove that the best approximate conic is 'unique'. Furthermore, We apply our characterization for the best geometric conic approximation to the degree reduction of cubic rational Bézier curve into quadratic one, to the conic offset curve approximation, and to the conic convolution curve approximation.

In Section 2, we explain the geometric properties for conic spline curves. In Section 3, we introduce a class of admissible curves, for which an error function and a normal distance to the plane curve $\mathbf{c}(s)$ are defined, and also characterize the best geometric conic approximation to the regular plane curve. In Section 4, we present numerical examples of three types for the geometric conic approximations: degree reduction, offset curve approximation and convolution curve approximation. In Section 5 , to verify our characterization for the best geometric conic approximation, we modify the nonlinear Chebyshev approximation theory and apply it to the family of error functions of conic approximations. In Section 6 , we summarize our works.

\section{Geometric properties for conic curves}

In this section we introduce the geometric properties of conic curves whose composite is called by conic spline. Let $\mathbf{b}(t), 0 \leq t \leq 1$, be the plane conic curve expressed by the standard quadratic rational Bézier form

$$
\mathbf{b}(t)=\frac{B_{0}^{2}(t) \mathbf{b}_{0}+w B_{1}^{2}(t) \mathbf{b}_{1}+B_{2}^{2}(t) \mathbf{b}_{2}}{B_{0}^{2}(t)+w B_{1}^{2}(t)+B_{2}^{2}(t)}, \quad 0 \leq t \leq 1,
$$

with (not collinear) control points $\mathbf{b}_{i} \in \mathbf{R}^{2}$, weights $w>0$ associated with $B_{1}^{2}(t)$, where $B_{i}^{2}(t), i=0,1,2$, are the Bernstein polynomials of degree $n=2$ defined by

$$
B_{i}^{n}(t)=\frac{n !}{i !(n-i) !} t^{i}(1-t)^{n-i}
$$

Thus any conic curve has degree of freedom seven, $\mathbf{b}_{0}, \mathbf{b}_{1}, \mathbf{b}_{2}$ and $w$. It is well known [13] that the tangent lines of $\mathbf{b}(t)$ at $t=0$ and 1 have 


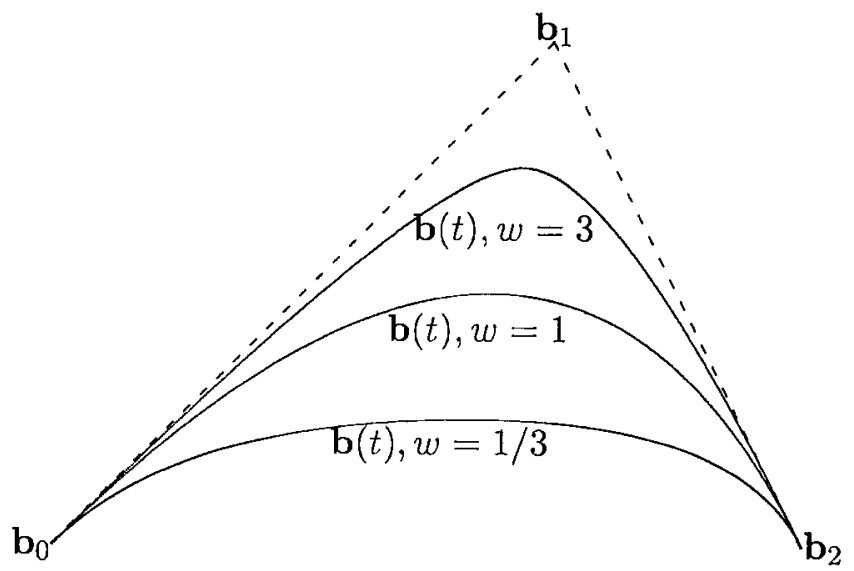

Figure 1 . The control polygon $\left[\mathbf{b}_{0} \mathbf{b}_{1} \mathbf{b}_{2}\right]$ is plotted by dash-lines and the associated conic curves $\mathbf{b}(t)$ having the weights $w=1 / 3,1$ or 3 , respectively, are plotted by connected lines.

the same directions of the lines $\mathbf{b}_{0} \mathbf{b}_{1}$ and $\mathbf{b}_{1} \mathbf{b}_{2}$, respectively, as shown in Figure 1. Also, the weight $w$ is called by fullness factor, because the more $w$ is large, the more the conic curve $\mathbf{b}(t)$ is close to the control polygon $\left[\mathbf{b}_{0} \mathbf{b}_{1} \mathbf{b}_{2}\right]$, as shown in Figure 1.

Let the conic curves $\mathbf{b}(t)$ and $\mathbf{p}(t)$ have the control polygons $\left[\mathbf{b}_{0} \mathbf{b}_{1} \mathbf{b}_{2}\right.$ ] and $\left[\mathbf{p}_{0} \mathbf{p}_{1} \mathbf{p}_{2}\right]$ with weights $w$ and $w^{\prime}$, respectively. The following propositions are well known in projective geometry $[13,19]$.

Proposition 2.1. If two conic curves $\mathbf{b}(t)$ and $\mathbf{p}(t)$ have five distinct intersection points, then $\mathbf{b}(t)=\mathbf{p}(t)$ for all $t \in I:=[0,1]$.

Proposition 2.2. Assume that two conic curves $\mathbf{b}(t)$ and $\mathbf{p}(t)$ have common end points and common tangent direction at both end points. Then $\left[\mathbf{b}_{0} \mathbf{b}_{1} \mathbf{b}_{2}\right]=\left[\mathbf{p}_{0} \mathbf{p}_{1} \mathbf{p}_{2}\right]$. Furthermore, $\mathbf{b}(t)$ and $\mathbf{p}(t)$ have another intersection point if and only if $\mathbf{b}(t)=\mathbf{p}(t)$, for all $t \in I$, i.e., $\left[\mathbf{b}_{0} \mathbf{b}_{1} \mathbf{b}_{2}\right]=$ $\left[\mathbf{p}_{0} \mathbf{p}_{1} \mathbf{p}_{2}\right]$ and $w=w^{\prime}$.

Supposed that the polygon $\left[\mathbf{b}_{0} \mathbf{b}_{1} \mathbf{b}_{2}\right]$ is fixed, and let $\Omega$ be the interior of the triangle $\triangle \mathbf{b}_{0} \mathbf{b}_{1} \mathbf{b}_{2}$. We define a mapping $h:(0, \infty) \times(0,1) \rightarrow \Omega$ by

$$
h(w, t):=\mathbf{b}(t)=\frac{B_{0}^{2}(t) \mathbf{b}_{0}+w B_{1}^{2}(t) \mathbf{b}_{1}+B_{2}^{2}(t) \mathbf{b}_{2}}{B_{0}^{2}(t)+w B_{1}^{2}(t)+B_{2}^{2}(t)}
$$

where $\mathbf{b}(t)$ is the conic curve with the control points $\mathbf{b}_{0}, \mathbf{b}_{1}, \mathbf{b}_{2}$, and weight $w>0$. Then the two variables function $h(w, t)$ is continuous and 
bijective from $(0, \infty) \times(0,1)$ onto $\Omega$. Thus the inverse map $h^{-1}$ is well defined on $\Omega$. Since the Jacobian determinant of $h(w, t)$ is nonzero at any point $(w, t) \in(0, \infty) \times(0,1)$, the map $h^{-1}$ is continuous at each point in $\Omega$ by the open mapping theorem for the two variables function $h$. Thus we have the following proposition which is needed to prove Theorem 5.5.

Proposition 2.3. Let $h(w, t)$ be the function defined as in Equation (2). Then $h$ is a homeomorphism from $(0, \infty) \times(0,1)$ onto $\Omega$.

\section{Characterization for the best geometric conic approxima- tion}

In this section we present the characterization for the best geometric conic approximation to the given regular plane curves. To high accurate approximate plane curves by polynomial or rational curves $[1,4,6,9$, $10,11,16,24]$, the parametrization-independent concept for contact of two curves at a common end point, also known as geometric continuity, is crucial in GHI (Geometric Herimte Interpolation).

Definition 3.1. ([11, 17]). Let $\mathbf{b}(t)$ and $\mathbf{c}(t)$ be $C^{k}$ regular parametric plane curves form I into $\mathbf{R}^{2}, k$ be a nonnegative integer and $t_{0}=0$ or 1 . Two curves $\mathbf{b}(t)$ and $\mathbf{c}(t)$ have contact of order $k$ at $t_{0}$ if there are $C^{k}$ reparametrizations $\tau_{1}$ and $\tau_{2}$ such that $\tau_{i}\left(t_{0}\right)=t_{0}(i=1,2)$, $\tau_{1}^{\prime}\left(t_{0}\right) \tau_{2}^{\prime}\left(t_{0}\right)>0$ and

$$
\left.\frac{d^{i}}{d t^{i}} \mathbf{b}\left(\tau_{1}(t)\right)\right|_{t=t_{0}}=\left.\frac{d^{i}}{d t^{i}} \mathbf{c}\left(\tau_{2}(t)\right)\right|_{t=t_{0}} \quad \text { for } i=0, \cdots k .
$$

If $\mathbf{b}(t)$ has contact of order $k$ to the curve $\mathbf{c}(s)$ at both end points, then $\mathbf{b}(t)$ is called by a $G^{k}$-approximation (or $G^{k}$-interpolation) to the curve $\mathbf{c}(s)$. In this paper the domains of curves are assumed to be $I$, which is possible using translation and scaling of the given domains. In this paper we characterize the best $G^{k}$ approximation to the given plane curve $\mathbf{c}(s)$ from all conic curves $\mathbf{b}(t)$ for $k=0$ or 1 . To find the approximate curve $\mathbf{b}(t)$, using the normal vector field, we define a certain class of admissible curves with respect to the curve $\mathbf{c}(s)$ for which the normal distance to $\mathbf{c}(s)$ can be defined as follows.

Definition 3.2. (See [11] and Figure 2). A curve $\mathbf{b}(t), 0 \leq t \leq 1$, is said to be admissible with respect to the curve $\mathbf{c}(s), 0 \leq s \leq 1$, if 


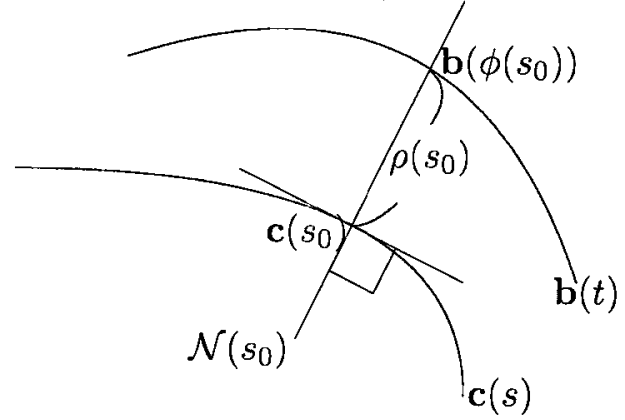

Figure 2. Admissible curve: the point $\mathbf{b}\left(\phi\left(s_{0}\right)\right), 0 \leq$ $s_{0} \leq 1$, lies on normal line $\mathcal{N}\left(s_{0}\right)$ of the source curve $\mathbf{c}(s)$ at $s=s_{0}$, and $\rho\left(s_{0}\right)$ is the signed distance from $\mathbf{b}\left(\phi\left(s_{0}\right)\right)$ to $\mathbf{c}\left(s_{0}\right)$.

(i) there exists a unique strictly increasing bijective map $\phi_{\mathrm{b}}: I \rightarrow I$ such that, for each $s \in I$, the point $\mathbf{b}\left(\phi_{\mathbf{b}}(s)\right)$ lies on the normal line $\mathcal{N}(s):=\left\{\mathbf{c}(s)+u \cdot \mathbf{n}_{\mathbf{c}}(s): u \in \mathbf{R}\right\}$ of $\mathbf{c}$ at $\mathbf{c}(s)$, where $\mathbf{n}_{\mathbf{c}}(s)$ denotes the unit normal vector of the plane curve $\mathbf{c}$ at $\mathbf{c}(s)$;

(ii) the tangent vector of $\mathbf{b}(t)$ at $t=\phi_{\mathbf{b}}(s)$ is not parallel to $\mathbf{n}_{\mathbf{c}}(s)$ for any $s \in I$.

Let $\mathcal{B}$ be the class of admissible conic curves with respect to the given plane curve $\mathbf{c}$, and let $\mathcal{B}_{k}:=\{\mathbf{b} \in \mathcal{B}: \mathbf{b}$ and $\mathbf{c}$ have contact order $k$ at the points $\mathbf{c}(0)$ and $\mathbf{c}(1)\}$, for $k=0,1$. Note that $\mathcal{B}_{1} \subset \mathcal{B}_{0} \subset \mathcal{B}$. If $\mathbf{b} \in \mathcal{B}$, then there exists a unique continuous map $\rho_{\mathrm{b}}: I \rightarrow \mathbf{R}$ satisfying:

$$
\mathbf{b}\left(\phi_{\mathbf{b}}(s)\right)=\mathbf{c}(s)+\rho_{\mathbf{b}}(s) \cdot \mathbf{n}_{\mathbf{c}}(s), s \in I .
$$

Since $\phi_{\mathbf{b}}(s)$ is surjective,

$$
\left\|\rho_{\mathbf{b}}\right\|_{\infty}=\max _{t \in I}\left(\min _{s \in I}\|\mathbf{b}(t)-\mathbf{c}(s)\|\right) .
$$

Hence we call $\left\|\rho_{\mathbf{b}}\right\|_{\infty}$ the normal distance (in $L_{\infty}$ sense) from conic $\mathbf{b}(t)$ to the plane curve $\mathbf{c}(s)$. If $\rho_{\mathrm{b}}(s)$ has the minimum norm among all $\mathbf{b} \in \mathcal{B}_{k}$, then $\mathbf{b}(t)$ is called the best approximation in $\mathcal{B}_{k}$ to the plane curve $\mathbf{c}(s)$.

Definition 3.3. A continuous function $g: I \rightarrow \mathbf{R}$ alternates (at least) $n$ times if and only if there are $n$ values $s_{i} \in I$ such that $0 \leq s_{1}<$ $\cdots<s_{n} \leq 1$ and $g\left(s_{i}\right)=-g\left(s_{i+1}\right)= \pm\|g\|_{\infty}, i=1, \cdots, n-1$. 5.

The following theorem is our main result, which is proved in Section 
TheOREM 3.4. For $k=0,1, \mathbf{b}(t)$ is the best $G^{k}$ conic approximation in $\mathcal{B}_{k}$ to the regular plane curve $\mathbf{c}(s)$ if and only if its signed error function $\rho_{\mathrm{b}}(s)$ alternates $4-2 k$ times. Furthermore, the best $G^{k}$ conic approximation to the curve $\mathbf{c}(s)$ is unique in $\mathcal{B}_{k}$.

REMARK. If a conic $\mathbf{b}(t)$ is in $\mathcal{B}$, then we have

$$
d_{H}(\mathbf{c}, \mathbf{b}) \leq\left\|\rho_{\mathbf{b}}\right\|_{\infty}
$$

where $d_{H}(\mathbf{c}, \mathbf{b})$ is the Hausdorff distance between two curves $\mathbf{c}$ and $\mathbf{b}$

$$
d_{H}(\mathbf{c}, \mathbf{b}):=\max \left(\max _{t \in I} \min _{s \in I}\|\mathbf{b}(t)-\mathbf{c}(s)\|, \max _{s \in I} \min _{t \in I}\|\mathbf{b}(t)-\mathbf{c}(s)\|\right),
$$

and the difference of two curves measured by the sense of human eyes is equal to their Hausdorff distance [8].

\section{Application and numerical examples}

In this section we present some applications of our characterization for the best geometric conic approximation. The applications consist of three examples which are most typical curve approximation problems in CAGD.

The first example is 'degree reduction' of the cubic rational Bézier curve into the quadratic one. Any plane cubic rational Bézier curve $\mathbf{c}(s), 0 \leq s \leq 1$, are defined by three cubic polynomials $x(s), y(s)$ and $w(s)$ such that $\mathbf{c}(s)=(x(s) / w(s), y(s) / w(s)), 0 \leq s \leq 1$, and it can be represented in Bézier form

$$
\frac{\sum_{i=0}^{3} w_{i} \mathbf{b}_{i} B_{i}^{3}(s)}{\sum_{i=0}^{3} w_{i} B_{i}^{3}(s)}
$$

where $\mathbf{b}_{i} \in \mathbf{R}^{2}$ and $w_{i}>0,0 \leq i \leq 3$, are control points and weights of the cubic rational Bézier curve $\mathbf{c}(s)$, and $B_{i}^{3}(s), 0 \leq t \leq 1$, are the cubic Bernstein polynomials defined in Equation (1). As shown in Figure 3, the cubic rational Bézier curve $\mathbf{c}(s), 0 \leq s \leq 1$, has the control points $(0$, $0),(1.7,0.2),(3.2,-0.1)$ and $(2.4,-0.9)$, and weights $1,1,1.3$ and 1 in order. The degree reduction makes an error in general. Thus using our characterization theorem, we obtain the best $G^{1}$ conic approximation curve $\mathbf{b}(t)$ to the cubic rational curve $\mathbf{c}(s)$. Then the conic curve $\mathbf{b}(t)$ has the control points $(0,0),(3.74,0.44)$ and $(2.4,-0.9)$ in order, and the weight $w=0.7475$. The Hausdorff distance between the two curves $\mathbf{b}(t)$ and $\mathbf{c}(s)$ is $3.14 \times 10^{-2}$ which is the minimal distance from all 


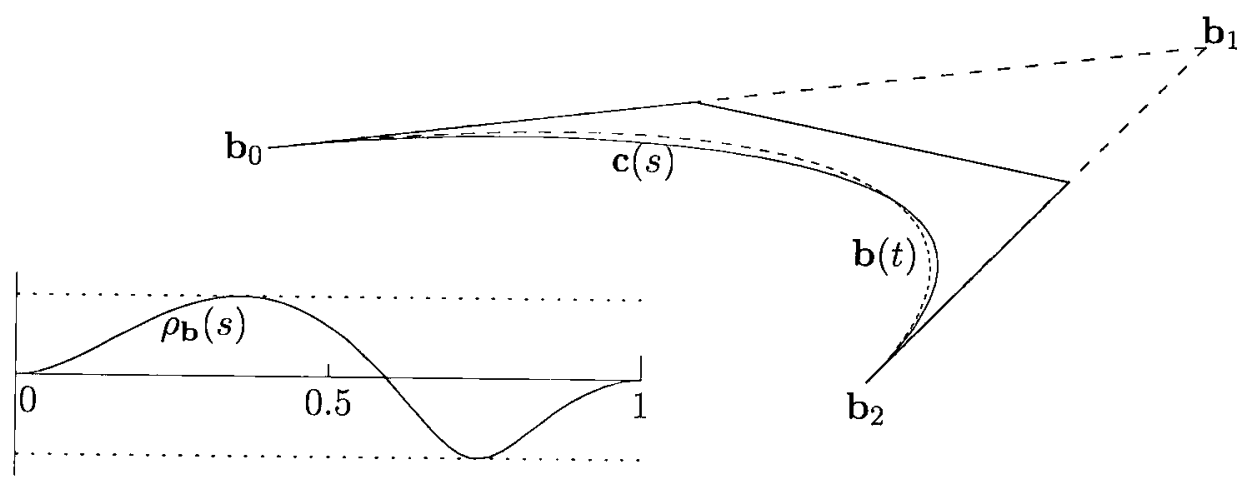

Figure 3. The given cubic rational Bézier curve $\mathbf{c}(s)$, $0 \leq s \leq 1$, and its control polygon are plotted by connected lines. The best $G^{1}$ approximate conic $\mathbf{b}(t)$ and its control polygon $\left[\mathbf{b}_{0} \mathbf{b}_{1} \mathbf{b}_{2}\right]$ are plotted by dash-lines. The sighed error function $\rho_{\mathrm{b}}(s)$ plotted in the left figure alternates twice.

$G^{1}$ conic curves to $\mathrm{c}(s)$. The signed error function $\rho_{\mathrm{b}}(s)$ alternates twice as shown in Figure 3. As the same method, we present another geometric conic spline approximation of the cubic rational spline which is the piece-wise cubic rational Bézier curves. As shown in Figure 4(a), the font " $\mathrm{r}^{\prime}$ is constructed by cubic rational spline, and its height is 540 units. Ahn[4] presented the optimal $G^{1}$ conic approximation with respect to the distance in a fixed direction and presented the maximum error bound 3.89 units. In this paper we can obtain the 'real' best $G^{1}$ conic spline approximation using our characterization theorem as shown in Figure 4(b) and obtain the maximum error bound 1.70 units.

The second example is geometric 'conic offset approximation'. ([12] or [15].) For the offset distance $d \in \mathbf{R}$, its offset curve $\overline{\mathbf{c}}_{d}: I \rightarrow \mathbf{R}^{2}$ of the conic curve $\mathbf{c}(s)$ is defined by

$$
\overline{\mathbf{c}}_{d}(s)=\mathbf{c}(s)+d \cdot \mathbf{n}(s),
$$

where $\mathbf{n}(s)$ is the normal vector of $\mathbf{c}$ at $\mathbf{c}(s)$ with outward direction of the conic curve. Using our characterization theorem, we find the best $G^{1}$ conic approximation $\mathbf{b}(t), 0 \leq t \leq 1$, to the offset curve $\overrightarrow{\mathbf{c}}_{d}(s), 0 \leq s \leq 1$. As an example, we consider a quartcr of ellipse with a $2: 1$ axes ratio in the first quadrant as shown in Figure 5(a). This can be represented by a conic curve $\mathbf{c}(s), 0 \leq s \leq 1$, with control points $(2,0),(2,1),(0,1)$, in order, 


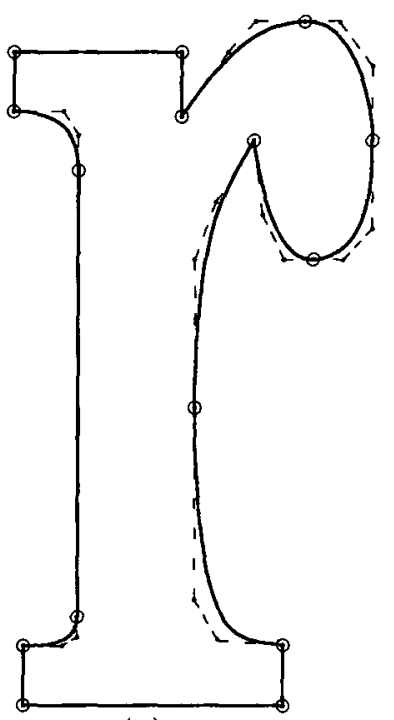

(a)

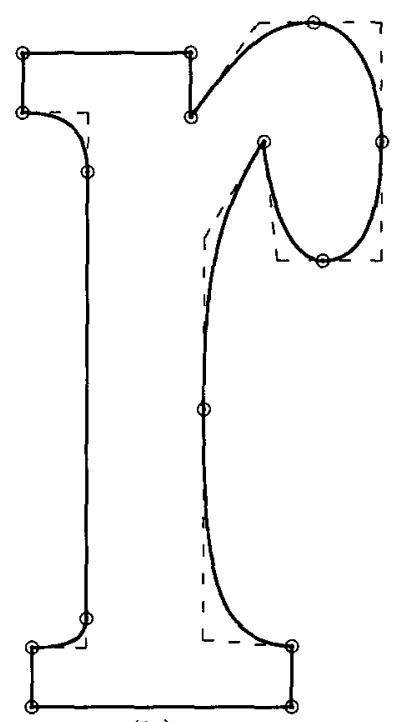

(b)

FiguRE 4. (a) The outline for the font " $\mathbf{r}$ " constructed by cubic rational spline plotted by thick lines. The polygon by dash-lines means the control-points of cubic rational spline curve and the circles are the junction points of two consecutive cubic segments. (b) The best $G^{1}$ conic spline approximation plotted by thick lines can be obtained using our characterization theorem. The polygon means the control-points of the conic spline curve in form of quadratic rational spline, and the circles are the junction points of the conic segments.

and weight $w=1 / \sqrt{2}$. ([13].) As shown in Figure 5(a), we obtain the best $G^{1}$ conic approximations $\mathbf{b}_{d}(t)$ to $\overline{\mathbf{c}}_{d}(s)$, for $d=1,2,3$, respectively. We also find the Hausdorff distances between the offset curve $\overline{\mathbf{c}}_{d}(s)$ and its approximation $\mathbf{b}_{d}(t)$ for $d=1,2,3$, using their signed error functions $\rho(s)$ for $d=1,2,3$, which alternates twice as shown in Figure 5(b).

The last example is geometric 'conic approximation of convolution curve' of any two conic curves. The convolution curve $\mathbf{c}_{1} * \mathbf{c}_{2}$ of two plane curves $\mathbf{c}_{1}(t)$ and $\mathbf{c}_{2}(s)$ is defined [5] by

$$
\left(\mathbf{c}_{1} * \mathbf{c}_{2}\right)(t)=\mathbf{c}_{1}(t)+\mathbf{c}_{2}(s(t))
$$

where the vector-valued derivative $\mathbf{c}_{1}^{\prime}(t)$ is parallel to $\mathbf{c}_{2}^{\prime}(s(t))$ and $\mathbf{c}_{1}^{\prime}(t)$. $\mathbf{c}_{2}^{\prime}(s(t))>0$ for a reparametrization $s=s(t)$. When $D_{1}$ and $D_{2}$ are 


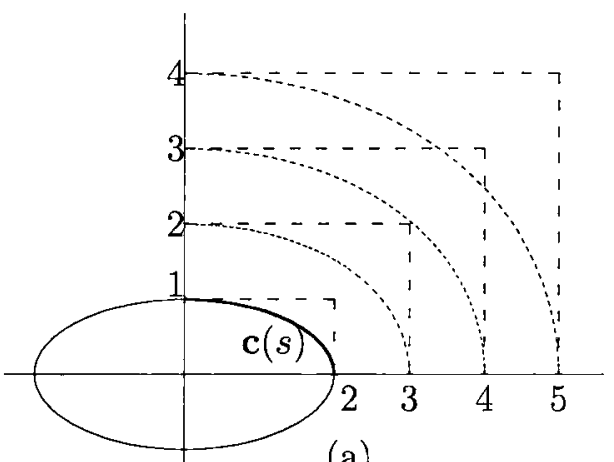

(a)

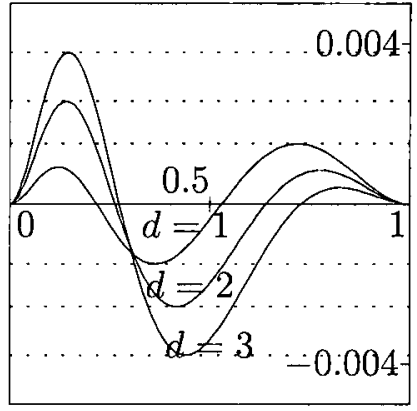

(b)

Figure 5. (a) A quarter of ellipse $\mathbf{c}(s)$ is plotted by thick lines, and the best $G^{1}$ conic approximation to the offset curves $\bar{c}_{d}(s), d=1,2,3$ are plotted by dash-lines. (b) The signed error functions $\rho(s), 0 \leq s \leq 1$, for $\bar{c}_{d}(s)$, $d=1,2,3$, alternate twice.

simply connected domain and have the boundary curves $\mathbf{c}_{1}$ and $\mathbf{c}_{2}$, respectively, the boundary of the Minkowski sum $D_{1} \oplus D_{2}$ is a subset of the convolution curve $\mathbf{c}_{1} * \mathbf{c}_{2}$ [5], where

$$
D_{1} \oplus D_{2}=\left\{\mathbf{x}_{1}+\mathbf{x}_{2}: \mathbf{x}_{1} \in D_{1} \text { and } \mathbf{x}_{2} \in D_{2}\right\} \text {. }
$$

Let $\mathbf{c}_{1}(t)$ be the ellipse which is an example of a shape of cross section of 'pen' as shown in Figure 6(a). The font " $\mathrm{D}$ " is produced by moving the center of ellipse along the curve $\mathbf{c}_{2}(s)$ in Figure 6(b), which consists of two conic and three straight lines. The Minkowski sum obtained by sweeping the curve $\mathbf{c}_{1}(t)$ along the curve $\mathbf{c}_{2}(s)$ is plotted roughly in Figure 6(c). As shown in Figure 6(d). the boundary of the Minkowski sum is the outlines for the font " $\mathrm{D}$ " and it consists of two parts: the convolution curves $\mathbf{c}_{1} * \mathbf{c}_{2}$ and subsets of ellipse. Finally, we approximate the convolution curves in Figure 6(d) by the best $G^{1}$ conic approximation as shown in Figure 6(e).

\section{Proof of the main theorem}

In this section we prove the main theorem (Theorem 3.4) using the alternation theorem of Rice[23]. The following definitions modified by Eisele [11] from the nonlinear approximation theory [7] are needed to prove the main theorem. Let $D$ be any nonempty subset of $\mathbf{R}^{n}$ and $F$ be any continuous mapping from $D \times I$ into $\mathbf{R}$. 


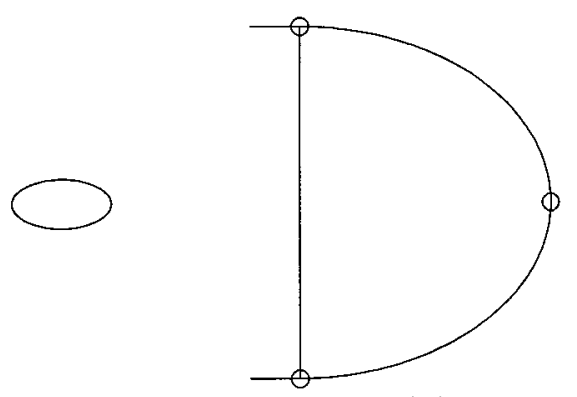

(a)

(b)

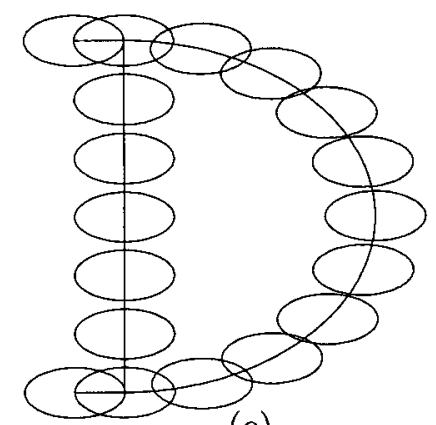

(c)

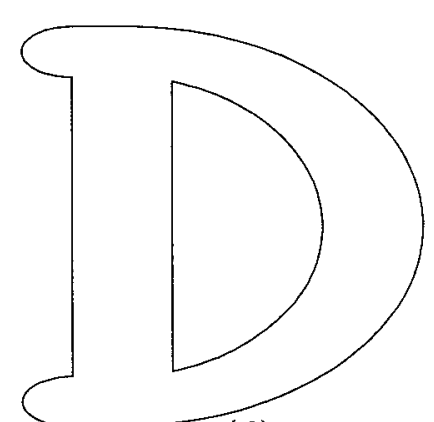

(d)

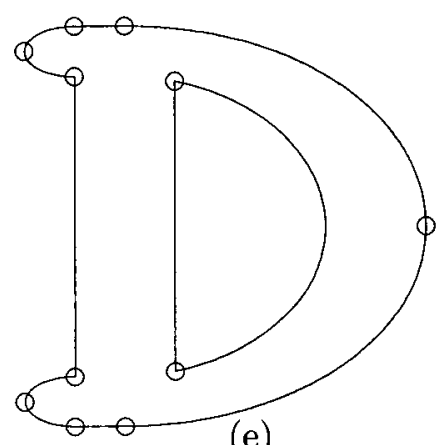

(e)

Figure 6. (a) An ellipse which is an example for a shape of cross section of "pen". (b) A skeleton curve for the font " $\mathrm{D}^{\prime}$ along which the center of the ellipse will be moved. (c) The Minkowski sum of two curves in (a) and (b). (d) The outline for the font " $\mathrm{D}^{\prime \prime}$ obtained from the boundary of the Minkowski sum. (e) The best $G^{1}$ conic spline approximation of the outline, which consists of seven conic curves and four straight lines.

Definition 5.1. $F$ is locally solvent of degree $m \in \mathbf{N}$ at $a \in D$ with respect to the interval $(0,1)$ if and only if given $m$ points $s_{j}$ with $0<s_{1}<$ $\cdots<s_{m}<1$ and $\varepsilon>0$ there is $\delta:=\delta\left(a, \varepsilon, s_{1}, \cdots, s_{m}\right)>0$ such that $\eta_{j} \in \mathbf{R}$ and $\left|\eta_{j}-F\left(a, s_{j}\right)\right|<\delta, j=1, \cdots, m$, imply the existence of $b \in D$ satisfying $F\left(b, s_{j}\right)=\eta_{j}, j=1, \cdots, m$, and $\|F(b, \cdot)-F(a, \cdot)\|_{\infty}<\varepsilon$. 
Definition 5.2. $F$ has the Property $Z$ of degree $m \in \mathbf{N}$ at $a \in D$ with respect to the interval $(0,1)$ if and only if for any $b \in D$, the difference $F(a, \cdot)-F(b, \cdot)$ either has at most $m-1$ zeros in the interval $(0,1)$ or vanishes identically.

DEFINITION 5.3. $F$ is varisolvent with respect to the interval $(0,1)$ if and only if at each point $a \in D$, both the local solvency and the Property $\mathrm{Z}$ with respect to $(0,1)$ are defined and have the same degree $m(a)$.

THeOREM 5.4. Let $f$ be a continuous function in $I$, and let $F$ be varisolvent with respect to $(0,1)$ with $F(a, 0)=f(0)$ and $F(a, 1)=f(1)$ for all $a \in D$. Then $F(a, \cdot)$ is the best approximation to $f$ if and only if $F(a, \cdot)-f$ alternates at least $m(a)+1$ times. Furthermore, if $F(a, \cdot)-f(\cdot)$ is not identically zero, there is at most one best approximation to $f$.

Proof. If $F(a, \cdot)-f$ is constant, then $F(a, s)-f(s)=F(a, 0)-$ $f(0)=0$ for every $s \in I$ and the statement is obvious. Thus we may assume that $F(a, \cdot)-f$ is not constant. Now the proofs of Theorem 3.9 and 3.10 in [7] carry over. Since $\|F(a, \cdot)-f\|_{\infty}$ has not its maximum at both 0 and 1 , the assertion follows.

Now, we prove the main theorcm. Let $\mathbf{b}(t)$ be the conic in $\mathcal{B}_{k}, k=$ 0,1 . Since the source curve $\mathbf{c}(s), 0 \leq s \leq 1$, and the approximate curve $\mathbf{b}(t)$ have the contact order $k \geq 0$ at both end points, we have $\mathbf{c}(0)=\mathbf{b}(0)$ and $\mathbf{c}(1)=\mathbf{b}(1)$, i.e., $\mathbf{b}_{0}=\mathbf{c}(0)$ and $\mathbf{b}_{2}=\mathbf{c}(1)$, where $\mathbf{b}_{0}, \mathbf{b}_{1}, \mathbf{b}_{2}$ are the control points of the conic $\mathbf{b}(t)$. Thus any conic $\mathbf{b}(t)$ in $\mathcal{B}_{0}$ is determined by three parameters $w>0, \mathbf{b}_{1}=\left(x_{1}, y_{1}\right)$, and any $\mathbf{b}(t)$ in $\mathcal{B}_{1}$ is determined by only one parameter $w>0$, since the vertex $\mathbf{b}_{1}$ is the intersection of the two tangent lines of $\mathbf{c}(s)$ at $s=0,1$. For $k=0,1$, let $\alpha:=\left(w, x_{1}, y_{1}\right) \in \mathbf{R}^{3}$ and let $\mathbf{b}_{\alpha} \in \mathcal{B}_{k}$ be the conic determined by weight $w$ and vertex $\mathbf{b}_{1}=\left(x_{1}, y_{1}\right)$. Let $A_{k} \subset \mathbf{R}^{3}$ be the set of all $\alpha$ such that $\mathrm{b}_{\alpha} \in \mathcal{B}_{k}$ for $k=0,1$. Note that $A_{1} \subset A_{0} \subset \mathbf{R}^{3}$. We define the mappings $\Phi: A_{k} \times I \rightarrow I$ and $E: A_{k} \times I \rightarrow \mathbf{R}$ by

$$
\Phi(\alpha, s):=\phi_{\mathrm{b}_{\alpha}}(s) \text { and } E(\alpha, s):=\rho_{\mathrm{b}_{\alpha}}(s), \quad(\alpha, s) \in A_{k} \times I .
$$

Note that $\Phi$ and $E$ are continuous. ([9]) We prove the following theorem using the inverse function theorem in Appendix.

Theorem 5.5. For $k=0,1$, and for $D:=A_{k} \subset \mathbf{R}^{3}$, the restricted mapping $F:=\left.E\right|_{(D \times I)}$ is locally solvent of fixed degree $3-2 k$ at each $\alpha \in D$ with respect to the interval $(0,1)$. 
Proof. See Appendix.

TheOREM 5.6. For $\alpha \in A_{k}, k=0,1$, the mapping $F:=\left.E\right|_{\left(A_{k} \times I\right)}$ has Property $Z$ at $\alpha$ of fixed degree $3-2 k$ with respect to the interval $(0,1)$.

Proof. Let $k=0$. If $F(\alpha, \cdot)-F\left(\alpha^{\prime}, \cdot\right)$ has three zeros in the open interval $(0,1)$, then it has five zeros in $I$, and the two conic curves $\mathbf{b}_{\alpha}$ and $\mathbf{b}_{\alpha^{\prime}}$ have five distinct common points. By Proposition 2.1, we have $\alpha=\alpha^{\prime}$ and $F(\alpha, \cdot)-F\left(\alpha^{\prime}, \cdot\right)=0$.

Let $k=1$. If $F(\alpha, \cdot)-F\left(\alpha^{\prime}, \cdot\right)$ has a zero in $(0,1)$, then $\mathbf{b}_{\alpha}$ and $\mathbf{b}_{\alpha^{\prime}}$ have a common point which is not the end points. It follows from Proposition 2.2 that $\alpha=\alpha^{\prime}$ and $F(\alpha, \cdot)-F\left(\alpha^{\prime}, \cdot\right)=0$.

Corollary 5.7. For $k=0,1, F:=\left.E\right|_{(A \times I)}$ is varisolvent of fixed degree $3-2 k$ with respect to $(0,1)$ in $A_{k}$.

Using the result of Theorem 5.4 and Corollary 5.7, we prove Theorem 3.4 .

Proof Theorem 3.4. For any $\alpha \in A_{k}, k \geq 0$, we have $\mathbf{b}_{\alpha} \in \mathcal{B}_{k}$ and $E(\alpha, 0)=E(\alpha, 1)=0$. It follows from Theorem 5.4 and Corollary 5.7 that $E(\alpha, \cdot)$ alternates $4-2 k$ times if and only if $E(\alpha, \cdot)$ is the best approximation in $A_{k}$ to the curve $f \equiv 0$, which means that $\mathbf{b}_{\alpha}$ is the best $G^{k}$ conic approximation in $\mathcal{B}_{k}$ to the curve c. By Equation (3), the assertion is obtained.

To show the uniqueness for the best approximation, we assume the existence of another best approximation $\mathrm{b}_{\alpha^{\prime}}$. By Theorem 5.4, $E(\alpha, \cdot) \equiv$ $E\left(\alpha^{\prime}, \cdot\right) \equiv 0$. Thus the Hausdorff distance between two conics $\mathbf{b}_{\alpha}$ and $\mathbf{b}_{\alpha^{\prime}}$ is zero. By Proposition 2.1, we get $\mathbf{b}_{\alpha}(t)=\mathbf{b}_{\alpha^{\prime}}(t)$ for all $t \in I$ and $\alpha=\alpha^{\prime}$.

\section{Comments}

In this paper we characterized the best $G^{k}$ conic approximation $\mathbf{b}(t)$, $0 \leq t \leq 1$, to the regular plane curve $\mathbf{c}(s), 0 \leq s \leq 1$, where $\mathbf{c}$ and $\mathbf{b}$ have contact order $k=0$ or 1 at both end points, and proved that the best approximate conic is unique. We applied our characterization to the degree reduction of cubic rational Bézier curve into quadratic one, conic offset curve approximation and conic convolution curve approximation, and presented the numerical results. 


\section{Appendix}

Proof of Theorem 5.5. Let there be given $\alpha_{0} \in A_{k}, \varepsilon>0$, and $3-2 k$ values $s_{j} \in(0,1)$ with $0<s_{1}<\cdots<s_{3-2 k}<1$. By Definition 5.1 and Equation (3), we have to find $\delta>0$ such that the equations $E\left(\alpha, s_{j}\right)=\eta_{j}, j=1, \cdots, 3-2 k$, have a solution $\alpha \in A_{k}$ with $\left\|E(\alpha, \cdot)-E\left(\alpha_{0}, \cdot\right)\right\|_{\infty}<\varepsilon$ if $\eta_{j} \in \mathbf{R}$ and $\left|\eta_{j}-E\left(\alpha_{0}, s_{j}\right)\right|<\delta$ for $j=1, \cdots, 3-2 k$. By the definition of $E$, we have to solve the $3-2 k$ equations (note that $E$ and $\Phi$ are not explicitly known):

(4) $\mathbf{b}_{\alpha}\left(t_{j}\right)=\mathbf{c}\left(s_{j}\right)+\eta_{j} \cdot \mathbf{n}\left(s_{j}\right)=:\left(u_{j}, v_{j}\right), \quad j=1, \cdots, 3-2 k$,

where $\alpha \in A_{k}$ and $t_{j} \in(0,1), j=1, \cdots, 3-2 k$ are unknown.

Since $E$ is continuous, and the set $\left\{\left(\alpha_{0}, s\right) \in \mathbf{R}^{4}: \alpha_{0}\right.$ is fixed in $A_{k}$ and $s \in I\}$ is compact in $\mathbf{R}^{4}$, there exists $\delta^{*}>0$ (depending only on $\varepsilon$ and $\alpha_{0}$ ) such that

$$
\left|E(\alpha, s)-E\left(\alpha_{0}, s\right)\right|<\varepsilon \quad \text { for all } 0 \leq s \leq 1
$$

if $\left\|\alpha-\alpha_{0}\right\|<\delta^{*}$. For simplicity of calculation, without loss of generality, we may assume that $\mathbf{b}_{0}$ is origin, $\mathbf{b}_{1}=\left(x_{1}, y_{1}\right), y_{1} \neq 0$, and $\mathbf{b}_{2}$ is the point $(1,0)$, using affine map in $\mathbf{R}^{2}$-plane. Then if $k=0,\left(x_{1}, y_{1}\right)$ and $w$ is undetermined, and if $k=1, w$ is undetermined.

First, let $k=0$. Using

$$
\mathbf{b}_{\alpha}(t)=\left(\frac{2 w x_{1} t(1-t)+t^{2}}{N(t)}, \frac{2 w y_{1} t(1-t)}{N(t)}\right)
$$

where $N(t):=B_{0}^{2}(t)+w B_{1}^{2}(t)+B_{2}^{2}(t)=(1-t)^{2}+2 w t(1-t)+t^{2}$, we have the following system 
(6) $G\left(w, x_{1}, y_{1}, t_{1}, t_{2}, t_{3}\right):=\left(\begin{array}{c}\left(2 w x_{1} t_{1}\left(1-t_{1}\right)+t_{1}^{2}\right) / N\left(t_{1}\right) \\ \left(2 w x_{1} t_{2}\left(1-t_{2}\right)+t_{2}^{2}\right) / N\left(t_{2}\right) \\ \left(2 w x_{1} t_{3}\left(1-t_{3}\right)+t_{3}^{2}\right) / N\left(t_{3}\right) \\ 2 w y_{1} t_{1}\left(1-t_{1}\right) / N\left(t_{1}\right) \\ 2 w y_{1} t_{2}\left(1-t_{2}\right) / N\left(t_{2}\right) \\ 2 w y_{1} t_{3}\left(1-t_{3}\right) / N\left(t_{3}\right)\end{array}\right)$

$$
=\left(\begin{array}{l}
u_{1} \\
u_{2} \\
u_{3} \\
v_{1} \\
v_{2} \\
v_{3}
\end{array}\right)
$$

where $G: \mathbf{R}^{6} \rightarrow \mathbf{R}^{6}$, which is equivalent to Equation (4). If $\eta_{j}:=$ $E\left(\alpha_{0}, s_{j}\right), j=1,2,3$, then $\left(\alpha_{0}, \Phi\left(\alpha_{0}, s_{1}\right), \Phi\left(\alpha_{0}, s_{2}\right), \Phi\left(\alpha_{0}, s_{3}\right)\right)$ solves Equation (7). This solution is denoted by $\mathbf{x}_{0} \in \mathbf{R}^{6}$.

We show that the Jacobian determinant of $G$ at $\mathbf{x}_{0}=\left(w, x_{1}, y_{1}, t_{1}, t_{2}, t_{3}\right)$ can be factorized as follows.

(7) $\operatorname{det}\left(\mathbf{D} G\left(\mathbf{x}_{0}\right)\right)=8 y_{1}^{2} w^{4}\left(t_{1}-t_{2}\right)\left(t_{2}-t_{3}\right)\left(t_{3}-t_{1}\right) \prod_{i=1}^{3} \frac{B_{1}^{2}\left(t_{i}\right)}{N\left(t_{i}\right)}$

Using simple calculations, we have

$$
\operatorname{det}\left(\mathbf{D} G\left(\mathbf{x}_{0}\right)\right)=w^{2} \prod_{i=1}^{3} \frac{1}{N\left(t_{i}\right)^{4}} \cdot \operatorname{det} \Gamma
$$

where $\Gamma$ is the $6 \times 6$ matrix

$$
\Gamma=\left(\begin{array}{cccccc}
B_{1}^{2}\left(t_{1}\right) M_{1}\left(t_{1}\right) & L\left(t_{1}\right) & 0 & K_{1}\left(t_{1}\right) & 0 & 0 \\
B_{1}^{2}\left(t_{2}\right) M_{1}\left(t_{2}\right) & L\left(t_{2}\right) & 0 & 0 & K_{1}\left(t_{2}\right) & 0 \\
B_{1}^{2}\left(t_{3}\right) M_{1}\left(t_{3}\right) & L\left(t_{3}\right) & 0 & 0 & 0 & K_{1}\left(t_{3}\right) \\
B_{1}^{2}\left(t_{1}\right) M_{2}\left(t_{1}\right) & 0 & L\left(t_{1}\right) & K_{2}\left(t_{1}\right) & 0 & 0 \\
B_{1}^{2}\left(t_{2}\right) M_{2}\left(t_{2}\right) & 0 & L\left(t_{2}\right) & 0 & K_{2}\left(t_{2}\right) & 0 \\
B_{1}^{2}\left(t_{3}\right) M_{2}\left(t_{3}\right) & 0 & L\left(t_{3}\right) & 0 & 0 & K_{2}\left(t_{3}\right)
\end{array}\right)
$$

and 


$$
\begin{aligned}
L(t) & :=B_{1}^{2}(t) N(t), \\
M_{1}(t) & :=x_{1}\left(2 t^{2}-2 t+1\right)-t^{2}, \\
M_{2}(t) & :=y_{1}\left(2 t^{2}-2 t+1\right), \\
K_{1}(t) & :=2 w x_{1}(1-2 t)+2 w t^{2}-2 t^{2}+2 t, \\
K_{2}(t) & :=2 w y_{1}(1-2 t) .
\end{aligned}
$$

By subtracting the $(3+i)$ th row times $K_{1}\left(t_{i}\right) / K_{2}\left(t_{i}\right)$ from $i$ th row for $i=0,1,2$ in $\Gamma$ and by $M_{1}(t)-M_{2}(t) K_{1}(t) / K_{2}(t)=y_{1} L(t) / K_{2}(t)$, we have

where

$$
\operatorname{det} \Gamma=\operatorname{det}\left(\begin{array}{c|c}
\Gamma_{1} & \mathbf{0} \\
\hline \Gamma_{2} & \Gamma_{3}
\end{array}\right)
$$

$$
\begin{aligned}
\Gamma_{1} & =\left(\begin{array}{lll}
y_{1} L\left(t_{1}\right) / K_{2}\left(t_{1}\right) & B_{1}^{2}\left(t_{1}\right) N\left(t_{1}\right) & -L\left(t_{1}\right) K_{1}\left(t_{1}\right) / K_{2}\left(t_{1}\right) \\
y_{1} L\left(t_{2}\right) / K_{2}\left(t_{2}\right) & B_{1}^{2}\left(t_{2}\right) N\left(t_{2}\right) & -L\left(t_{2}\right) K_{1}\left(t_{2}\right) / K_{2}\left(t_{2}\right) \\
y_{1} L\left(t_{3}\right) / K_{2}\left(t_{3}\right) & B_{1}^{2}\left(t_{3}\right) N\left(t_{3}\right) & -L\left(t_{3}\right) K_{1}\left(t_{3}\right) / K_{2}\left(t_{3}\right)
\end{array}\right) \\
\Gamma_{2} & =\left(\begin{array}{ccc}
B_{1}^{2}\left(t_{1}\right) M_{2}\left(t_{1}\right) & 0 & L\left(t_{1}\right) \\
B_{1}^{2}\left(t_{2}\right) M_{2}\left(t_{2}\right) & 0 & L\left(t_{2}\right) \\
B_{1}^{2}\left(t_{3}\right) M_{2}\left(t_{3}\right) & 0 & L\left(t_{3}\right)
\end{array}\right) \\
\Gamma_{3} & =\left(\begin{array}{ccc}
K_{2}\left(t_{1}\right) & 0 & 0 \\
0 & K_{2}\left(t_{2}\right) & 0 \\
0 & 0 & K_{2}\left(t_{3}\right)
\end{array}\right) .
\end{aligned}
$$

Note that $\operatorname{det} \Gamma=\operatorname{det} \Gamma_{1} \cdot \operatorname{det} \Gamma_{3}$ and $\operatorname{det} \Gamma_{3}=K_{2}\left(t_{1}\right) K_{2}\left(t_{2}\right) K_{2}\left(t_{3}\right)$. Thus

$$
\operatorname{det} \Gamma=-y_{1} \operatorname{det} \Gamma_{1}^{\prime} \prod_{i=1}^{3} L\left(t_{i}\right)
$$

where

$$
\Gamma_{1}^{\prime}:=\left(\begin{array}{ccc}
B_{1}^{2}\left(t_{1}\right) & K_{2}\left(t_{1}\right) & K_{1}\left(t_{1}\right) \\
B_{1}^{2}\left(t_{2}\right) & K_{2}\left(t_{2}\right) & K_{1}\left(t_{2}\right) \\
B_{1}^{2}\left(t_{3}\right) & K_{2}\left(t_{3}\right) & K_{1}\left(t_{3}\right)
\end{array}\right) .
$$

By a simple calculation of determinant of $3 \times 3$ matrix, we obtain

$$
\operatorname{det} \Gamma_{1}^{\prime}=-8 w^{2} y_{1}\left(t_{1}-t_{2}\right)\left(t_{2}-t_{3}\right)\left(t_{3}-t_{1}\right)
$$

Therefore, Equation (7) follows from Equations (8)-(10).

Since $s_{i} \neq s_{j}$ for $i \neq j$, and $y_{1} \neq 0$, the Jacobian determinant $\operatorname{det}\left(\mathbf{D} G\left(\mathbf{x}_{0}\right)\right)$ in Equation (7) is nonzero. By the implicit (or inverse) function theorem there exist sufficiently small neighborhoods $U$ of $\mathbf{x}_{0}$ and $V$ of $G\left(\mathbf{x}_{0}\right)$ such that $G: U \rightarrow V$ is one to one and onto, and 
such that if $\mathbf{x} \in U$, then $\left\|\mathbf{x}-\mathbf{x}_{0}\right\|<\delta^{*}$. Thus we can find a suffciently small $\delta>0$ such that if $\left|\eta_{j}-E\left(\alpha_{0}, s_{j}\right)\right|<\delta$ for $j=1,2,3$, then $\mathbf{y}:=\left(u_{1}, u_{2}, u_{3}, v_{1}, v_{2}, v_{3}\right) \in V$, where $u_{j}, v_{j}$ are obtained from Equation (4), by using the estimate

$$
\left\|\mathbf{y}-G\left(\mathbf{x}_{0}\right)\right\| \leq\left\{\sum_{j=0}^{3}\left|\eta_{j}-E\left(\alpha_{0}, s_{j}\right)\right|^{2}\right\}^{1 / 2}<\sqrt{3} \delta .
$$

Since $G: U \rightarrow V$ is an onto mapping, there exists $\mathbf{x}=\left(\alpha, t_{1}, t_{2}, t_{3}\right):=$ $G^{-1}(\mathbf{y}) \in U$ which solves Equation (7) and satisfies Equation (4)

$$
\mathbf{b}_{\alpha}\left(t_{j}\right)=\mathbf{c}\left(s_{j}\right)+\eta_{j} \cdot \mathbf{n}\left(s_{j}\right)
$$

and $\left\|E(\alpha, \cdot)-E\left(\alpha_{0}, \cdot\right)\right\|_{\infty}<\varepsilon$ because $\left\|\alpha-\alpha_{0}\right\|<\left\|\mathbf{x}-\mathbf{x}_{0}\right\|<\delta^{*}$.

Now, let $k=1$. Since $\alpha \in A_{1}$, then $\mathbf{b}_{1}=\left(x_{1}, y_{1}\right)$ is fixed and so is $\Omega$. There exists a sufficiently small $\delta>0$ such that $\left|\eta_{1}-E\left(\alpha_{0}, s_{1}\right)\right|<\delta$ implies the existence of $\alpha:=\left(w, x_{1}, y_{1}\right)$ and $t_{1}$ satisfying

(i) $\left\{\mathbf{c}\left(s_{j}\right)+\eta_{j} \cdot \mathbf{n}\left(s_{j}\right)\right\} \subset \Omega$ for all $j$;

(ii) $\mathbf{b}_{\alpha}\left(t_{1}\right)=\mathbf{c}\left(s_{1}\right)+\eta_{1} \cdot \mathbf{n}\left(s_{1}\right)$ (see [13]);

(iii) $\left\|\alpha_{0}-\alpha\right\|<\delta^{*}$ by Proposition 2.3 .

Since (ii) is equivalent to $E\left(\alpha, s_{1}\right)=\eta_{1}$ and $\left\|\alpha_{0}-\alpha\right\|<\delta^{*}$ implies Equation (5), we get the assertion.

ACKNOWLEDGEMENTS. The author is very grateful to the Editor and anonymous referees for the valuable comments.

\section{References}

[1] Y. J. Ahn and H. O. Kim, Approximation of circular arcs by Bézier curves, J. Computational and Applied Mathematics 81 (1997), 145-163.

[2] - Curvatures of the quadratic rational Bézier curves, Computers and Mathematics with Applications 36 (1998), no. 9, 71-83.

[3] Y. J. Ahn, H. O. Kim, and K. Y. Lee, $G^{1}$ arc spline approximations of quadratic Bézier curves, Computer-Aided Design 31 (1998), 615-620.

[4] Y. J. Ahn, Conic Approximation of planar curves, Computer-Aided Design (to appear).

[5] C. Bajaj and M. S. Kim, Generation of configuration space obstales: The case of moving algebraic curves, Algorithmica 4 (1989), no. 2, 157-172.

[6] C. de Boor, K. Höllig, and M. Sabin, High accuracy geometric Hermite interpolation, Computer Aided Geometric Design 4 (1987), 169-178.

[7] D. Braess, Nonlinear Approximation Theory, Springer, Berlin, 1986.

[8] M. G. Cox and P. M. Harris, The approximation of a composite Bézier cubic curve by a composite Bézier quadratic curve, IMA J. Numerical Analysis 11 (1990), 159-180. 
[9] W. L. F. Degen, Best approximations of parametric curves by spline, In T. Lyche and L.L. Schumaker, editors, Mathematical Methods in CAGD and Image Processing, pp. 171-184, Newyork, 1992, Academic Press.

[10] — High accurate rational approximation of parametric curves, Computer Aided Geometric Design 10 (1993), 293-313.

[11] E. F. Eisele, Chebychev approximation of plane curves by splines, J, Approximation Theory 76 (1994), 133-148.

[12] G. Farin, Curvature continuity and offsets for piecewise conics, ACM Trans. Graph. 8 (1989), no. 2, 8999.

[13] _ Curves and Surfaces for Computer Aided Geometric Design, Academic Press, San Diego, CA, 1993.

[14] R. T. Farouki, Analysis of the offset to a parabola, Computer Aided Geometric Design 12 (1995), 639-646.

[15] _ , Conic approximation of conic offsets, J. Symbolic Computation 23 (1997), no. 3, 301-313.

[16] M. Floater, An $\mathcal{O}\left(h^{2 n}\right)$ Hermite approximation for conic sectons, Computer Aided Geometric Design 14 (1997), 135-151.

[17] J. A. Gregory, Geometric continuity, In T. Lyche and L.L. Schumaker, editors, Mathematical Methods in CAGD, pp. 353 371, Nashville, 1989, Academic Press.

[18] H. J. Kim and Y. J. Ahn, Good degree reduction of Bézier curves using Jacobi polynomials, Computers and Mathematics with Applications 40 (2000), 1205 1215.

[19] E. T. Lee, The rational Bézier representation for conics, In geometric modeling : Algorithms and new trends, pp. 3-19, Philadelphia, 1987, SIAM, Academic Press.

[20] D. S. Meek and D. J. Walton, Approximating smooth planar curves by arc splines, J. Computational and Applied Mathematics 59 (1995), 221-231.

[21] T. Pavlidis, Curve fitting with conic splines, ACM Trans. Graph. 2 (1983), 1-31.

[22] T. Pratt, Techniques for conic splines, In Proceedings of SIGGRAPH 85, pp. 151-159. ACM, 1985.

[23] J. K. Rice, The Approximation of Functions, Vol.II - Advanced Topics, AddisonWesley, London, 1969.

[24] R. Schaback, Planar curve interpolation by piecewise conics of arbitrary type, Constructive Approximation 9 (1993), 373389.

Department of Mathematics

Ajou University

Soowon, Kyunggi-do 442-749, Korea

E-mail: ahn@madang.ajou.ac.kr 